\title{
Hypertension and depression — the terrible two
}

\author{
Päivi Korhonen
}

University of Turku, Finland

Arterial Hypertens. 2016, vol. 20, no. 4, pages: 198-199

DOI: $10.5603 / A H .2016 .0021$

Depression is one of the most common mental health disorders globally, presenting an increasing burden everywhere - as are hypertension-related diseases. Depression nearly doubles the risk for developing coronary heart disease, and in those already suffering from heart disease, depression nearly doubles the risk of mortality [1, 2]. This elevated cardiac risk associated with depression is independent of classical risk factors, and similar in degree to smoking and hypercholesterolaemia.

The mechanisms whereby depressive illness can trigger atherosclerosis are supposed to be deregulation in the sympathetic nervous system and hypothalamic-pituitary-adrenal axis [3, 4]. This in turn has a number of harmful downstream effects, including increased heart rate and reduced heart rate variability, endothelial dysfunction, vasoconstriction, production of inflammatory cytokines, platelet activation, insulin resistance, and elevated risk for development of hypertension and metabolic syndrome.

There are many standardized questionnaires to assess depressive symptoms, but the diagnosis requires a clinical interview and fulfilment of at least four depressive symptoms. Alternatively to questionnaires, a preliminary assessment of depression can be made with two core questions:

- Do you feel down, depressed and hopeless?

- Have you lost interest and pleasure in life?

Giving a "yes" answer to either one of these core questions may be as effective as using longer screening instruments [5].
Major depression is more prevalent in people who have suffered a major cardiac event; the large European EUROASPIRE Study showed rates with up to $35 \%$ in men and $65 \%$ in women [6].

If we look at figures from the community, we see rates of depression of $10 \%$ in general practice clinics [7]. There are two European studies conducted among hypertensive patients in which the diagnosis of depression has been made by clinical interview - "the state of the art method". In the Norwegian community-based study, the prevalence of depression was $6 \%$, while in the hospital-based study in the Netherlands, the prevalence was $11 \%$ [8].

Depression may act as a barrier to the adoption of healthy lifestyle and to its maintenance. There are a number of behavioural and lifestyle factors which are present in depressive patients that can increase the chance of developing hypertension and cardiovascular disease. These include increased rates of smoking, alcohol intake, physical inactivity, and obesity [9]. Major depression also predicts poor adherence to medications [10].

It is important to recognize depression in hypertensive subjects and when recognized, to treat it. It is not known which of the various antidepressants available is the best to lower the risk for cardiovascular events. However, selective serotonin reuptake inhibitors can be regarded as the first choice because of their good tolerability and safety profile also in the cardiac setting. On the contrary, tricyclic antidepressants and monoamine oxidase inhibitors may have

\footnotetext{
Address for correspondence:

Päivi Korhonen Professor of General Practice, University of Turku,

Finland Joukahaisenkatu 3-5A

20014 University of Turku, Finland

e-mail: paikor@utu.fi
}

V M Copyright () 2016 Via Medica, ISSN 2449-6170 
toxic cardiac side effects, and these drugs should be avoided in those who have comorbid cardiac conditions.

Luckily, a Cochrane review has indicated that moderate aerobic exercise in the order of $30 \mathrm{~min}$ utes five times a week is beneficial in relieving depressive symptoms with similar efficacy to cognitive behavioural therapy [11] — with an apparent help to control blood pressure, too.

When prescribing beta-blockers, physicians may have concerns about development of depression. However, this conventional wisdom is not supported by randomized trial data. Depression, fatigue and sexual dysfunction are common complaints also among placebo treated subjects.

Depression is often invisible, silent, and denied. But it is common in hypertensive subjects. The toxic combination of hypertension and depression leads to poor health outcomes for both conditions. Depression should be considered as a modifiable risk factor for hypertension and cardiovascular disease - just like we do for smoking, and hypercholesterolaemia. Depression should be screened at least among uncontrolled hypertensive subjects, and busy clinicians could utilize the core questions as a quick and simple screening method for the terrible two - hypertension and depression.

\section{References}

1. Katon W.J., Lin E.H., Russo J. et al. Cardiac risk factors in patients with diabetes mellitus and major depression. J. Gen. Intern. Med. 2004; 19: 1192-1199.

2. Nicholson A., Kuper H., Hemingway H. Depression as an aetiologic and prognostic factor in coronary heart disease: a meta-analysis of 6362 events among 146538 participants in 54 observational studies. Eur. Heart J. 2006; 27: 2763-2774.

3. Esler M., Turbott J., Schwarz R. et al. The peripheral kinetics of norepinephrine in depressive illness. Arch. Gen. Psychiatry 1982; 39: 295-300.

4. Veith R.C., Lewis N., Linares O.A. et al. Sympathetic nervous system activity in major depression: basal and desipramine-induced alterations in plasma norepinephrine kinetics. Arch. Gen. Psychiatry 1994; 51: 411-122.

5. U.S. Preventive Services Task Force. Screening for depression in adults: U.S. Preventive Services Task Force Recommendation Statement. Ann. Intern. Med. 2009; 151: 784-792.

6. Kotseva K., Wood D., De Backer G. et al. EUROASPIRE III: a survey on the lifestyle, risk factors and use of cardioprotective drug therapies in coronary patients from 22 European countries. Eur. J. Cardiovasc. Prev. Rehabil. 2009; 16: 121-137.

7. Cassano P., Fava M. Depression and public health: an overview. J. Psychosom. Res 2002; 53: 849-576.

8. Li Z., Li Y., Chen L., Chen P. Prevalence of depression in patients with hypertension. Medicine 2015; 94: e1317.

9. Stapelberg N.J., Neumann D.L., Shum D.H., Mcconnell H., Hamilton-Craig I. A topographical map of the causal network of mechanisms underlying the relationship between major depressive disorder and coronary heart disease. Aust. N. Z. J. Psychiatry 2011; 45: 351-369.

10. Gehi A., Haas D., Pipkin S., Whooley M.A. Depression and medication adherence in outpatients with coronary heart disease: findings from the Heart and Soul Study. Arch. Intern. Med. 2005; 165: 2508-2513.

11. Cooney G.M., Dwan K., Greig C.A. et al. Exercise for depression. Cochrane Database Syst. Rev. 2013; 9: CD004366. doi: 10.1002/14651858.CD004366.pub6. 